\title{
Deep Conventionalism about Evolutionary Groups
}

\author{
Matthew J. Barker, Concordia University \\ Joel D. Velasco, California Institute of Technology
}

Concepts of evolutionary groups are some of the most important concepts in biology and its philosophy. These groups include often-cited players in evolutionary processes, such as populations, species, biological races, and lineages of various sorts. In a broad sense, certain products of evolution are also considered evolutionary groups, including clades of species, of populations, of organisms, and of gene families. Assumptions about evolutionary groups feature in nearly every biological study, whether explicitly evolutionary, molecular, or otherwise. And philosophers have exported views about evolutionary groups as far afield as debates about how we should organize and fund science in democratic societies. ${ }^{1}$

The widespread importance of concepts of evolutionary groups helps make disputes about them important. But it makes perhaps even more important a rare consensus. The consensus is a form of objectivism about what determines which collections are evolutionary groups. It allows that our research interests may help determine which group concept is best in a given case. But it says that on any single prevailing group concept, we as minded agents do not help fix or determine which candidate groups are indeed evolutionary groups under that concept; instead, objective facts alone suffice for that. Although a mix of biological, chemical, psychological, physical facts and so on may be recognized among these objective facts, it is harmless in this 
context to use "biological facts" to refer them. What is important is that this set includes only mind independent facts. It excludes by definition those facts that are instead fully or partially mind dependent, e.g., facts about our research interests, perceptual abilities, general values, and so on. Given these terms, the consensus view says objective biological facts alone suffice-we as minded agents are not needed-to determine whether a candidate group is in fact a kind of evolutionary group.

Explicit statements of objectivism about evolutionary groups in biology literatures are typically each about one or another specific kind of evolutionary group. And fellow biologists seldom challenge these. When molecular phylogeneticists and developmental botanists argue that the AGL6-like family of genes is a clade that has existed for at least 300 million years, colleagues may dispute whether the AGL6-like group really is a clade. ${ }^{2}$ But the vast majority on either side of any such dispute will agree that it is the biological facts alone that determine whether the AGL6-like group satisfies the notion of clade that they all (let us suppose) are using. In another chapter of the objectivist consensus, evolutionary ecologists argue that many a biological taxon has objective cohesion owing to gene flow between but not beyond the populations constituting it. ${ }^{3}$ Again, any disputes about this will very probably not indict objectivism. Indeed, objectivism about evolutionary groups is typically taken for granted without explicit statement. And when stated, authors happily leave it as an assumption. ${ }^{4}$ What could be more obvious than, say, that a clade of plants would be a clade even were we never here to discover that? 
Philosophers have more explicitly treated or adopted objectivism about evolutionary groups as a general consensus, rather than dwelling only on more specific objectivisms about this or that kind of group. For example, Dupré, Ereshefsky, and Kitcher clarify that their respective pluralisms about biological classification are consistent with objectivism about many kinds of evolutionary groups (though they may disagree on some kinds of groups). ${ }^{5}$ But their discussions do not aim for, and so understandably do not provide, close scrutiny or detailed defense of objectivism about evolutionary groups. The basic and assumed idea is that many different evolutionary groups are, despite their differences, similarly objective because the evolutionary processes that involve and produce such groups operate objectively.

The sway the consensus holds in both local chapters and as a whole is remarkable. Objectivism about clades lies behind the common view that there is a single universal tree of life. Objectivism about taxonomic groups prevails among even non-objectivists about taxonomic ranks, and is part of the idea that any one species concept univocally classifies organisms (barring vagueness) despite competing species concepts ambiguously cross-classifying them. ${ }^{6}$ Authors working on the Human Genome Diversity Project have used population objectivism to justify decisions about what kind of informed consent to acquire and when, and about which research methods and data to use. ${ }^{7}$ And the objectivist consensus has motivated attempts in more general philosophy of science to retain a form of scientific realism despite recognizing an increasing number of ways in which values (in a general sense) must shape scientific inquiry. ${ }^{8}$ 
Despite its dominance, we will argue that this consensus is mistaken because objectivism about many and perhaps all commonly recognized kinds of evolutionary groups is mistaken. This paper aims to displace the consensus with a new view, Deep Conventionalism.

This new view consists of two parts. The first is a pluralism that is deeper than familiar pluralist views attributed to Dupré, Kitcher, and Ereshefsky. Unlike their pluralisms, ours undermines the objectivism of the consensus. The second part of our view fills this void with a conventionalism that applies to a wide variety of evolutionary groups. This conventionalism says that even given any single, specific evolutionary grouping concept, typically something more than the objective biological facts must determine or fix which things are such groups. The "something more" is a mix of facts about us. The mix includes various conventions of ours, but also our research interests, values, abilities, and so on. We use "conventionalism" for short.

To proceed, we first situate Deep Conventionalism among related views. This positions us to clarify a key notion of suppressed variables and the deep pluralism associated with these. We then undertake the central task of showing how such variables ensure that our view holds for a variety of evolutionary grouping concepts, using cohesive functional units, populations, and clades as exemplars. Finally, we 
discuss potential objections and highlight implications for a range of important positions.

\section{Situating Deep Conventionalism}

What is an evolutionary group? An innocuous answer is that an evolutionary group is any group of things that have certain evolutionarily salient relations that set them apart from other things. Exactly when things enjoy such relations, they make an evolutionary group out of what would otherwise have been, from an evolutionary perspective, a mere group or collection.

The category of "evolutionary group" divides into distinct kinds of evolutionary groups. Authors recognize these with definitions and elaborations of different evolutionary grouping concepts, distinguished by appeal to different evolutionarily salient relations. For instance, "species" is said to name one kind of evolutionary group, and "population" another. Definitions of the species concept typically attempt to identify the evolutionarily salient relations between organisms and/or groups of them in virtue of which those things together form a species. Definitions of "population" typically attempt to identify the relations in virtue of which organisms form a population.

Sometimes a dispute about a group concept is about which definition of it is "correct", "best" or "legitimate"-about which identified relations are the ones that make a group a species, or a population, etc. This is sometimes further understood as 
competition between more specific concepts, each vying to be the specification of the broader concept under dispute, the one that objectively does or does not apply to each candidate group. Someone with this understanding sees the biological species concept, phylogenetic species concepts, and ecological species concepts battling to be the objective species concept.

What we will call pluralistic objectivism is an increasingly popular way of interpreting these sorts of disputes differently, a way of qualifying or even eliminating some of the dispute in each case while still conforming to the objectivist consensus. ${ }^{9}$ This pluralism and how it differs from our own is most easily seen by drawing from its application in the species concepts literature, though when later arguing for our own view we will only treat species concepts implicitly, through discussions of other related important kinds of evolutionary groups.

Pluralistic objectivism has two noteworthy features. First, it claims that the concept in question either subsumes or should be eliminated in favor of two or more-a plurality—of finer-grained concepts, each of which is legitimate. This pluralism about legitimacy allows that two concepts thought to be competing for legitimacy are instead each legitimate for distinct purposes. For example, the biological species concept is said to be legitimate (or the best, or the correct concept to use) for some purposes, phylogenetic species concepts for others, and ecological ones for still others. Our interests help determine which finer-grained concept of a given broader type is best in which case. 
Second, pluralistic objectivism says that for each finer-grained concept it is objective facts alone that suffice to determine how a set of things divides into groups under that concept. So our interests help determine which of these concepts to use in a given case, but the objective facts have already determined what the groups are under each of the concepts our interests are choosing between.

More precisely, take the set of organisms, $S$, consisting of two populations of organism on opposites sides of a mountain, population North and population South. According to pluralistic objectivism, objective facts suffice to determine whether and how $S$ divides into biological species groups, or just biological groups when these are deemed objective but their assignment to the species rank is not, and whether and how it divides into ecological species groups, or just ecological groups. Suppose the pluralistic objectivist believes that objective facts determine that North and South together form one biological species group, while objective facts also determine that North is one ecological species group, and South another. That is, one finer-grained concept lumps North and South, the other splits them. Then, in (say) a research study or classification project involving $S$, our interests enter the picture, helping determine whether it is best or legitimate for us to recognize the lumping divisions of the biological species concept, or the splitting divisions of ecological ones, or both, or neither. Independently of us, the divisions are there. 
In typical empirical conditions our view disagrees with the objectivism in pluralistic objectivism. There are many ways to convey this, with some ways useful to some people and others to others. Our view says that conventions-facts about our interests, values, abilities, and so on-help determine not only which concept is relevant or legitimate in a given case, but also to which candidate groups it applies in that case. Our conventions are needed along with objective facts to fix the extensions of evolutionary group concepts. Conventions are needed to determine whether a collection of organisms is a population, clade, give type of species, etc. How many biological species are in a given set of organisms is not fully fixed by objective facts across all research contexts; in some research contexts facts about us pair with objective facts to give one count, in other research contexts they pair to give others. Conventions help determine not only significance but also accuracy of group identify (and associated taxonomic) claims. In typical empirical conditions, the biological facts cannot determine whether North and South form distinct ecological species, let alone species simpliciter.

As we will clarify near the end of the paper when discussing concept splitters, our conventionalism about grouping concepts, including finer-grained ones, should not motivate pluralistic objectivists to simply split their finer-grained concepts into even finer-grained ones in the hope of reaffirming objectivity at still finer conceptual levels. We are stuck with our conventionalism and should abandon pluralistic objectivism. Our reasoning begins in the next section by clarifying what we call Indeterminacy Pluralism. This is pluralism with respect to the values that can be 
taken by the suppressed variables associated with any single prevailing evolutionary grouping concept, not pluralism about multiple concepts being legitimate. To understand suppressed variables, we start with a non-biological, linguistic example. But we stress that this is only to intuitively convey the form that Indeterminacy Pluralism takes, and how it can mandate conventionalism. We will then have to show that the biological cases take this form. Distant views in philosophy of language do no work in any of this.

\section{Suppressed Variables}

Suppose Charles is at a large picnic with much of Alfred's extended family. Alfred is in a small group of people around a punch bowl, and Charles, walking towards them, senses that the small group is not enjoying the live country music. But the rest of the people at the picnic love the music. Charles asks, "So is this small group of you unified in your response to country music?"

Alfred answers "yes." But this is correct only by drawing on context to further specify the question. Alfred gathers that Charles asked his question with certain kinds of responses in mind, and certain kinds of country music. Without explicitly or implicitly choosing particular values for these variables, there is no correct answer to the question. And on other values of the variables, we can imagine that the relevant facts ensure that Alfred's answer is instead not correct. 
Take the case in which the small punch bowl group includes just Alfred and his brother and sister. For the kinds of response variable, choose the "emotional response" value. For the kinds of country music variable, choose the "pop-country music" value. Then, given facts about his family, Alfred can assure you that he was correct to affirm that the small punch bowl group is unified in its response to country music. He and his siblings each react with disgust to pop-country music, and more so than any of the attending extended family does. However, now change the value of the kinds of country music variable to "alt-country music." Then Alfred's affirmative answer to Charles's question switches to not correct. Alfred likes alt-country music and his brother loves it. But his sister detests it, more than any people in the extended family. Changing the other variable, from "emotional response" to "sensory-motor response," may also make Alfred's affirmative answer incorrect.

In cases like the picnic scenario, semantic facts about the meaning of "response to country music" leave many variables open. Short of further inputs, there is no semantic fact of the matter about whether the kinds of response variable takes the "emotional response" value or "sensory-motor response" value. Given that such variables do often get fixed in the face of these factual shortfalls, something else must add to the semantic facts to fix the variable values.

In the picnic case, that "something else" is pretty clearly our conventions about contextual information. Suppose that at the picnic it is pop-country music, in particular, that is playing when Charles asks his question. Then very probably, both 
he and Alfred have in mind the "pop-country music" value of the kinds of country music variable. And this is most likely because both of them are following a reasonable convention, which here implies: if it is pop-country music that is playing at the picnic, then presume that the kind of country music that the question is about is pop-country music. Indeed, it seems that in cases with conditions like this case, conventions must help with any fixing of variable values.

The relevant biological variables, not just linguistic ones, are also of this kind and lead to similar results. To see this, first consider that in the picnic case we have Indeterminacy Pluralism consisting in two conditions. One: whether a group of people is unified in its response to country music depends on variables that can each take one of a plurality of values that are all included among the facts. In fact, Alfred emotionally responds to alt-country music in one way, and to pop-country in another. Two: for some or all of these variables some different available values would on their own lead to incompatible results, e.g., to the punch bowl group having a unified response on some variable values but not on others. So the facts independent of our contributions leave it indeterminate whether the punch bowl group is unified in its response to country music. Given that indeterminacy in some cases like this is overcome, our contributions are needed to make up those indeterminacy shortfalls.

Analogously for prevailing kinds of evolutionary groups, Indeterminacy Pluralism is true and concerns the plurality of values that are available for variables of being an evolutionary group of the given kind. Regardless of whether there is a plurality of 
legitimate species concepts as familiar pluralisms claim, the above two conditions are typically met when using any one of these or any other prevailing evolutionary group concepts. And again we must make up this shortfall conventionally. To make good on these claims, the next three sections discuss prominent examples of forward looking evolutionary groups and then backward looking evolutionary groups; for brevity, general objections are discussed after these sections rather than repeated in each.

\section{Functional Units and Cohesion}

Many evolutionary groups are what Baum calls "functional units", characterized by "cohesion or causal efficacy" that allows them to be "players" or forward looking groups in ongoing evolutionary processes (op. cit., p. 74). Although authors, including Baum, typically have species in mind when discussing these units, some note that the cohesion that is supposed to make species functional units is also had to greater degrees by some non-species groups, such as populations, and to lesser degrees by other non-species groups, such as multi-species syngameons and perhaps some higher taxa. ${ }^{10}$ We dwell first on the species grade of this cohesion: species cohesion.

Species cohesion has been important in many articulations of the nature of species since the Modern Synthesis. ${ }^{11}$ This is explicit in some species concepts, such as the evolutionary species concepts of Wiley and Simpson, and implicit in others, such as Mayr's biological species concept. ${ }^{12}$ Species cohesion is also important to various interventional and field studies, e.g., attempts to explain why conspecific populations together trace a distinct trajectory through the space of evolutionary pressures, 
including various forms of natural selection. Some such projects attempt to discover and mathematically represent relationships between effective population sizes, population subdivision, migration, and species cohesion. For instance, a traditionally recognized relationship is that the effective number of migrants, $N_{e} m$, from one population to another must be $\geq 1$ for "maintaining species cohesion" across those populations..$^{13}$ Studies of evolutionary forces attempt to refine this view. ${ }^{14}$ Although the importance of species cohesion and similar sorts of functional cohesion differ from the importance of the clades in phylogenetics, many phylogeneticists insist that species are special precisely because of their functional cohesion. ${ }^{15}$

The question for us is whether species cohesion is a conventional sort of unity due to featuring suppressed variables. Only recently have authors provided the clarification of "species cohesion" required to answer this. ${ }^{16}$ Species cohesion is a grade of evolutionary response cohesion that involves organisms or populations responding similarly to evolutionary pressures. Importantly, whether a group responds in such a way depends partially on the contrast class. Take a collection of populations. It manifests evolutionary response cohesion exactly when the responses of its populations to evolutionary pressures are more similar to each other than to any outside the collection. This is for a collection to be exclusive, in at least one way, among others. Without this particular relativization to things outside the collection, it is hard to see how the collection could have the cohesion that is supposed to set it apart from other things - give it functional unity. 
Once it is clear that evolutionary response cohesion distinguishes evolutionary groups that we call functional units, it is easy to see that being such a unit depends on the values that suppressed variables take. These are variables of evolutionary response cohesion. Recall populations North and South, flanking the mountain. They will face many evolutionary pressures, often concurrently: a drought, a nutrient deficiency, emergence of an advantageous mutation. And there are different responses they can have to any one pressure: this trait declines in frequency in one population and increases in the other; that trait increases in both populations. Minimally then, two suppressed variables of evolutionary response cohesion (of any grade) that can take many values are which evolutionary pressures and which aspects of response.

In typical cases, there will be an enormous number of values these variables can take because organisms and populations have many traits and face many evolutionary pressures. On many combinations of these values the two mountain populations would count as having evolutionary response cohesion while on many others, they would not. Suppose that in each population, just 1\% of organisms have a suite of genes that, during depressed humidity, contribute to their retaining moisture far better than the other $99 \%$ of organisms. Then there is a series of devastating droughts. The suite of genes increases to $35 \%$ representation in both populations. In organisms of other nearby populations, genes involved in moisture retention are quite variable, resulting in no pattern of frequency response during the droughts. Choosing "moisture retention genes" for the which aspects of response variable, and 
"series of droughts" for the which evolutionary pressures variable, along with many other values of these variables that similarly relate the populations, would count the two mountain populations as having associated evolutionary response cohesion. The responses of moisture retention genes in those two populations are more similar to each other than to any responses in other populations.

At the same time, in North, and in all populations nearby except South, a new sequence at a genetic locus has emerged that dramatically helps utilize increased sunlight hours for energy production. Spikes in sunlight hours accompany the droughts. Selection then facilitates a spike in population frequencies of the new sunlight utilization sequence-except in South, which does not yet enjoy that sequence. If we change the value of the which aspects of response variable, from "water retention genes," to "sunlight utilization locus" plus other aspects of response that similarly relate all the populations, then the two mountain populations would not count as having evolutionary response cohesion.

This clarifies how functional units distinguished by evolutionary response cohesion will typically satisfy the two conditions of Indeterminacy Pluralism. To help verify that this is typically so, most any study of population differentiation will do. Barbará et al. (op. cit.) recently described a nice model for studying population differentiation across continental radiations. The model involves populations of Alcantarea species, perennial plants in Brazil that grow on large granite outcrops (similar to Ayers Rock, aka Uluru). Populations in these species made a useful model partly because 
measurements suggested that factors known to complicate some population differentiation studies (e.g., populations diverging markedly from Hardy-Weinberg and selection/drift equilibriums) were absent, or otherwise would not significantly distort assessments of these populations.

Highly varied traits characterized organisms in these populations. For example, all eight microsatellite loci investigated in populations of one species, Alcantarea imperialis, "were polymorphic, with up to 14 alleles per locus" (ibid., p. 1985). And the scattering of populations across granite outcrops suggests varied evolutionary pressures across those populations. Together these points indicate there are many values that the variables responses to evolutionary pressures and which aspects of response will take across the studied populations of Alcantarea imperialis (first condition of Indeterminacy Pluralism). Also, evidence suggested that for at least some of these variables some different available values would on their own lead to incompatible verdicts on whether the populations of the Alcantarea imperialis jointly manifest the species grade of evolutionary response cohesion (second condition of Indeterminacy Pluralism). Genetic distances between populations of Alcantarea imperialis, for example, were sometimes nearly as large as between that species and another Alcantarea species (ibid., p. 1986). Genetic variance, too, between conspecific populations was near what it was between the species (ibid., p. 1988), and many researchers believe that in many cases variance between conspecific populations is even greater than that between species. These statistical measures of distance and variance strongly suggest that many particular genetic responses to evolutionary 
pressures are more similar between populations of distinct species than between conspecific populations.

Generally across functional unit candidates, many of the biological values available for suppressed variables of evolutionary response cohesion would count the candidate as being a functional unit. Many other available biological values would have the opposite result. Both results cannot obtain. And the biological facts do not choose which of all the biological values are taken by the variables. We must do that. Species cohesion and other grades of evolutionary response cohesion are therefore conventional sorts of unity in light of the Indeterminacy Pluralism that is true of them. This entails conventionalism about functional units distinguished by such cohesion.

\section{Populations and Interaction Rate Exclusivity}

Not all forward looking functional units are distinguished by some grade of evolutionary response cohesion. For others, it is how they causally interact with each other, rather than how they causally respond to shared evolutionary pressures, that makes them functional units of an evolutionary kind. ${ }^{17}$ Populations are the prime example.

Millstein usefully compares prevailing distinct population concepts in terms of permissiveness. ${ }^{18}$ Some are astonishingly permissive, recognizing any collection of organisms within a species as a population (ibid., p. 61). For our purposes it would be 
most convincing to show that the least permissive, or most specific, population concept that is common in evolutionary studies features Indeterminacy Pluralism. Millstein, following in the wake of others, refines the definition of such a concept. Roughly, "the causal interactionist population concept" says that a population is any group of multiple conspecific organisms that is the largest group for which the internal rates of survival and reproduction interactions are much higher within the group than outside it (ibid., p. 67).

As with evolutionary response cohesion, the evolutionary group-making property that this definition picks out is a kind of unity or exclusivity property. It is relativized to things outside candidate populations, as you would expect of a property that is supposed to unify and set apart a group from other things. In this case, it is survival and reproduction interaction rates that are supposed to be distinctive between group members, relative to outsiders. Effectively these interaction rates are to be greater between group members than between them and outsiders.

This property also features Indeterminacy Pluralism due to variables that can take many values, some large sets of which would suggest a group has the property and other large sets of which would imply otherwise. We find these variables at more than one level. At a first level, there is a variable that is not suppressed at all, the kind of interaction variable. It is not suppressed because two values of this variable "survival interaction" and "reproduction interaction" - are explicitly referenced in the description of the definitive property. These two values can pull in opposite 
directions. Many organisms frequently interact with others in a way that changes their life expectancy (e.g., negatively in the case of direct or indirect competition, and positively in the case of cooperation), without changing their expected reproductive output (ibid.). The situation escalates if we omit the stipulated restriction of a population to members of the same species, as Godfrey-Smith suggests we do to properly understand natural selection, and as one must (on pain of circularity) if one defines "species" in terms of populations. ${ }^{19}$ Highest rates of reproductive interactions for some plant in my garden might connect it with pollinators and seed dispersers, while highest rates of survival interactions might connect it with other plants crowding it for soil and sun.

One level down we find two suppressed variables: kinds of survival interaction and kinds of reproductive interaction. These can take several values, indicated when Millstein notes there are several different kinds of survival and reproductive interactions, respectively (op. cit., pp. 67-68). Among the reproductive kind, she cites successful matings, unsuccessful matings, and different offspring rearing activities. Survival interactions include direct competition, indirect competition, and cooperation. Values for each of these will often simultaneously pull in opposite directions with respect to a candidate group's being "interaction rate exclusive." A tree in Mauro's backyard has perennially poor fruit. The local deer nearly always choose the neighbor's tree fruit instead. Furthermore, most of the fruit from Mauro's tree rots below it, leaving seeds to struggle for the little light penetrating through other crowding trees of Mauro's. The struggling seeds of Mauro's tree involve that 
tree in frequent (unfavorable) reproductive interactions with Mauro's other trees; the fruit of that tree involve it in frequent (unfavorable) reproductive interactions with the neighbor's tree. Many organisms frequently each have many reproductive interactions, some of which suggest connections to one group, some to another, others to another still, and so on. Likewise for their survival interactions.

Suppose we accept that for many a candidate population in the popular sense that Millstein refines, many values for the variables we have discussed would imply that the candidate has the exclusivity or unity that marks such populations. And many other values would imply the candidate does not have this property. Then we again have Indeterminacy Pluralism, and many population boundaries must be ones we help fix. Populations popularly conceived are then conventional in our sense.

\section{Clades, Splitting and Genealogical Exclusivity}

In many areas of biology the central evolutionary grouping concept is that of a clade or a monophyletic group. Clades are evolutionary groups because they feature a kind of evolutionary unity - they are united by a shared common ancestry, which makes them backward looking groups. Relative recency of common ancestry often explains why members of a clade share the traits that they do, grounds a variety of inferences about the past, and provides evidence about what unseen traits in members of the group will be like. Such features make clades so important in taxonomy that a common view of biological taxa is that they must be clades. The importance extends far beyond taxonomy. Phylogenetic trees are recognized as the background 
information required for a huge number of inferences and explanations. But trees are simply a representation of which groups under examination form clades that do the real explanatory work. Essentially any question in evolutionary biology, or other branches of biology that make evolutionary assumptions, depends on history and so on clades. $^{20}$

But in fact there is no single "common ancestry" relationship that grounds clade groupings. A standard definition of "clade" is that it is some species and all of its descendants. Yet it is not clear at all which groups are species. (Nor clear if there are any species. ${ }^{21}$ ) Further, some of the most popular views about species require that they are clades, and so at least those views cannot define "clade" in terms of species. For these reasons, it is now common to see clades defined directly in terms of groups of populations or organisms and their relationships. ${ }^{22}$ But there are different, incompatible ways of understanding the history of populations and of organisms. Take these in turn.

Defenses of phylogenetic concepts of species often talk about trees of populations, to argue that all taxa (including species) should be monophyletic groups of populations. ${ }^{23}$ That is, a clade should be some ancestral population and all of its descendants. This maneuver avoids talking about ancestral species, and avoids having delineation of clades depend on delineation of speciation events. But we then replace the avoided problem with the problem of delineating populations and population lineage splits. Velasco argues that lineage splits are context-dependent. ${ }^{24}$ One rough 
argument for this is that lineage splits represent a loss of cohesion between groups and the introduction of distinct evolutionary paths. However for certain kinds of traits a group may still be cohesive, while for others, the very same group may be broken up into independent trajectories. Only the context and associated conventions can determine which kinds of traits are of interest and so must help determine whether a lineage split has occurred.

The history of populations is naturally "loose" in a way that allows for some reticulation between groups. The very idea of migration dictates that it must be possible to have some gene flow between distinct populations without thereby collapsing them. How much reticulation is allowed is precisely what is at issue and what drives the point that lineage-splitting (and so cladehood) is context-dependent. Grant and Grant talk about distinct clades of Darwin's finches and place them on a phylogenetic tree, but later discuss hybridization between these groups. ${ }^{25}$ There are many reasons to treat sister species of Darwin's finches as distinct clades. But whether the relevant lineages should be considered separate at all depends on context and convention.

This brings us to the history of organisms, because for some purposes, in some contexts, we want to be strict, and then it is important to think of clades as genealogically exclusive groups of organisms. That is: a group of organisms, all of which are more closely related to each other than to any organisms outside the group, with no exceptions such as hybrids. De Queiroz and Donoghue introduced this 
concept of exclusivity to the taxonomic literature to separate it from monophyly in reticulating groups (such as organisms within a single species). ${ }^{26}$ But there are different ways of understanding how organisms are related to one another. Baum and Shaw first carefully spelled out exclusivity in terms of genetic concordance, but Velasco defines it in terms of organismal parent-offspring relationships..$^{27}$ These two kinds of group are incompatible, with some biological projects concerned with one and different projects the other. ${ }^{28}$

Thus when we ask whether a group is genealogically exclusive, there is a suppressed variable that we might call kind of genealogical exclusivity. It can take (at least) the values "recency of organismal common ancestry" or "genetic concordance." But the biology alone does not determine which of these values the variable takes. So long as the available values are objectively incompatible as these two often are, any determination of whether a candidate group is genealogically exclusive is determination that we help with. This is because in such a typical case, our research interests, conventions, and so on, are involved in selecting among the available variable values. These contributions of ours must help select, if variable values are taken at all. Genealogical exclusivity is therefore conventional in our broad sense determined by biology plus by us. When being a clade is being genealogically exclusive, we also help determine whether something is a clade.

We do not always want our understanding of common ancestry to be as strict as genealogical exclusivity, even though that exclusivity represents a kind of shared 
ancestry that can ground many kinds of inferences. After all, a small number of hybrids between two different clades destroys either kind of genealogical exclusivity just described. And often we want to understand the distribution of some "broader level" feature such as biogeography, in which case it seems appropriate to think of the history of whole populations as determined by population lineage splits. But in these cases conventions help fix the variable value "distinct population lineage" in place of "being genealogically exclusive." And we saw that this fixed value itself has deeper suppressed variables, because population splits depend on contexts that have incompatible outcomes and which the biological facts alone do not choose between. So at multiple levels there is Indeterminacy Pluralism and conventionalism.

The general source of this is that different parts of a taxon have different histories. Which parts we care about varies across contexts. Our research interests help decide between the looser "population lineage" definition of clade or the more strict "genealogical exclusive group of organisms" idea. What is important to see is that on either of these readings, there are still further suppressed variables whose objective values would incompatibly dictate which things are population level lineages or which organisms are most closely related to each other. And the biological facts leave us with a plurality of possible values that lead to incompatible grouping of organisms into clades. Further details are needed for any determination of cladehood.

This is most obvious in extreme cases like Thermotogales. While much of the group's history remains uncertain, ribosomal RNA and other "core" operational genes give us 
strong reason to believe that the Thermotogales are a bacterial group that share a "cellular" history with the bacteria Aquifilales; however, the majority of their genome indicates some other phylogenetic position - including many genes which are clearly of archaeal origins. ${ }^{29}$ Context combined with various conventions helps determine whether Thermotogales is a clade of Bacteria, a clade of Archaea, or not a clade at all. While Thermotogales is among the most extreme cases we know, this kind of context dependence is unavoidable. There is then is no unique objective grouping of organisms into clades and so no uniquely correct tree of life.

\section{6. $\quad$ Lumpers and Splitters}

For splitters, those with a preference for finer-grained concepts, an objection now quickly comes to mind. Just as Dupré, Ereshefsky and Kitcher have split "species" into "genealogical species" and "interbreeding species" and others, objectivists can split the concept of "clade" into "population level clade" and "organism level clade". This may concede conventionalism about "clade", but relocates objectivism to the finergrained concepts that the splitting of "clade" produces. Perhaps this splitter strategy can be applied to "functional cohesive unit" and "population" too.

Our above discussion indicates that the "clade" splitter's first division, between "population level clade" and "organism level clade," will only confront the further suppressed variables we have uncovered for each of these. Can the splitter then simply try and split yet again? Technically, yes. But consider the kinds of concepts that result: 
- $\quad$ "population level clade" as clarified by a strict-but-not-too-strict "with $<1$ incoming migrant per generation per population"

- $\quad$ organism level clade as defined by being an exclusive group due to being a clade on the plurality of the genome tree with respect to all genes and all organisms"

Putative objectivism about these concepts faces two problems. The first is that key components of these are also conventional. It is doubtful that biological facts alone fix what counts as a generation or a migrant, for example, much less what it takes to fix what counts as an organism or a population.

The second and more decisive problem is that these concepts, and the additional splitting that produced them, are theoretical dead ends. These concepts are not evolutionary grouping concepts at all. They are ad hoc constructions for the sole purpose of being objective and would play no role in biological theorizing. Being a clade is important. If we want to know what a clade is, we should focus on the role that the term "clade" plays in biological theory. But if we do this, it is clear that being a clade is tied up with many different kinds of processes, patterns, and methods of detection, and is fundamentally intertwined and interdefined with other "problem" concepts like lineage, species, and population. Being overly precise in defining "clade" robs the term of its power to play the large number of roles that it is expected to play.

This problem is even clearer for splitting "functional cohesive unit," which would give way to concepts such as: 
- "functionally cohesive group with respect to trait T1 and pressure P1"

- " "functionally cohesive group with respect to trait T2 and pressure P2," and...

If one of these applies to a group, it will typically apply to only that group-the one featuring T1 that is subject to P1, for example. Such concepts do not pick out kinds to which many member groups belong and over which theoretically interesting generalizations and predictions hold. Splitting "population" yields similarly vapid concepts:

- $\quad$ population due to rate exclusivity with respect to survival interactions S1 and reproductive interactions R1"

- $\quad$ population due to rate exclusivity with respect to survival interactions S2 and reproductive interactions R2"

The theoretical reasons for caring about the kinds of groups we have focused on in this paper are absent for those produced when avoiding our conventionalism by splitting. Better to not split and retain conventional concepts that are theoretically important. In other words, the utility of evolutionary group concepts depends on their flexibility in application. Empirical facts about the organization and diversity of the biological world dictate that our grouping concepts allow for flexibility in application in distinct contexts. It would be a mistake to conclude that this diversity requires instead a tremendous explosion in the number of evolutionary grouping concepts that we must use. 
Lumpers will agree that splitting buys objectivity at too high a theoretical cost, and that we should retain instead the theoretically important concepts we have analyzed. But many lumpers will keep their agreement with us short, insisting that these unsplit concepts can objectively apply with theoretical significance across many contexts. For example, of course we would allow that in one context a group of populations is a functional cohesive unit if all their trait responses to all evolutionary pressures are more similar to each other than to any other populations. But the lumper argues that we also need to allow that a different group of populations can have the same property of being a functional cohesive unit with just $80 \%$ of their trait responses to all evolutionary pressures being more similar to each other than to any other population. In another context, 75\% may suffice if this includes the right traits. In still others the difference may concern percentage of pressures instead of traits, but we still have a cohesive functional unit. We may need to recognize similar flexibility to being a population: in one context, exclusivity with respect to large sets or certain reproductive and survival interactions suffices, in another a smaller set of a different but especially salient mating interactions is enough. Perhaps a similar kind of consideration could apply to the different kinds of genealogical connections relevant to being a clade.

Effectively, these suggestions indicate that the unsplit concepts of "functional cohesive unit," Millstein's "population" and "clade" are all cluster concepts. ${ }^{30}$ This is regardless of whether a particular group that one of these concepts picks out is a natural kind, an individual, or something else-in each case, the concepts are defined 
by disjunctive clusters of conditions; no one of these conditions is necessary for application of the concept, but a variety of combinations of them is each sufficient.

There are two candidate ways to claim biological facts alone determine when such concepts apply. One is by putatively objective weighting schemes. Such a scheme hopes to tell us how much different factors matter in different cases. Notice that the weighting scheme that says that all factors matter equally across all cases in all contexts is still a weighting scheme (it is a strictly equitable one). The problem with any of these schemes is not that they are hopeless; they can be quite useful. The problem is for an objectivist reading of them. Thinking that biological facts alone determine which traits matter to what degree in what context is hopeless. Using the language of suppressed variables, our arguments in this paper have already implied these weightings are conventionally determined.

This fact inspires the second candidate way to claim that biological facts alone determine when cluster concepts apply in particular cases. The idea is that cluster grouping concepts cannot be defined in terms of the relative similarities or kinds of interactions between things that groups comprise. Granted, the disjunctive specifications of conditions above may be epistemic guides-they may help indicate whether a given concept applies to a group. But these conditions obtaining between things grouped are not what makes those groups evolutionary ones. Instead, the groups themselves have ontological priority as real units, whether as individuals (the populations of Millstein) or natural kinds (the species taxa of Boyd, op. cit., and 
Wilson, Barker and Brigandt, op. cit.). Reductive definitions of them are then bound to fail and the ontologies of evolutionary groups resist deeper specification.

This "groups first" view may seem a desperate way for the objectivist lumper to avoid the conventionalism of weighting schemes. But it is a difficult view to refute. We think it becomes more plausible when elements of it are retained in a conventionalist framework. In that framework, we appeal to biological facts, but also draw on our research interests, abilities, values, and so on, to conventionally determine which things are evolutionary individuals or kinds. Indeed, the least strained descriptions of biological practice accord with this. After conventional "group first" delineations pick out the groups, epistemically useful cluster specifications can be given and explain why these groups, rather than other possible groupings, fall under the relevant concept.

\section{Conclusion and Broader Issue}

Our chief conclusion is that evolutionary grouping concepts, such as those of clades, functional cohesive units, and populations, do not objectively delineate groups, but rather, when they do apply to some collection or organisms, they must do so with the help of our conventions. More specifically, facts about the context and our conventions, interests, and values combine with the biological facts to determine whether some collection of organisms is a group of a particular kind. The central reason for this concerns the suppressed variables built into these concepts. The concepts apply only when the variables take values. But the variables cannot take 
values independently of context. And without our conventions helping to determine which values are taken, there is simply no fact of the matter about which collections are groups according to that concept. For just a flavor of how this conclusion is relevant not only to discussions of evolutionary groups, but also connects to a range of other issues, we finish with snapshots of broader implications.

The common assumption that the evolutionary groups we study form objectively determined branches on a single objective tree of all life is false. Prevailing taxonomic concepts each do not unambiguously divide sets of organisms into taxa when taking only objective biological facts as inputs. Instead, in different contexts, different groups are properly regarded as taxa.

Biological taxonomies have featured as case studies in broad "science and values" debates about whether and how our conventions and interests do or must attach to such taxonomies. Some objectivists concede that our conventions and interests help determine the significance of taxonomies, but we now see this is not a sufficient concession. Conventions and interests also help determine the accuracy of those taxonomies, because conventions and interests help fix the groups taxonomized.

Objectivity of evolutionary groups is often thought to contribute to or feature as a part of policy justifications. For instance, to help justify the conservation of certain caribou groups, the Committee on the Status of Endangered Wildlife in Canada (COSEWIC) can be understood as appealing to normative principles of the following 
sort: if a described group of organisms objectively satisfies the definition of an evolutionary group concept (even a definition arbitrarily chosen among competitors), then the group is a unit of diversity that should be protected or conserved when endangered. ${ }^{31}$ Even though the justification of this principle will probably be normative, it applies to any given group only if that group is objective. Policy makers, including COSEWIC, often suggest that the only or main obstacle to satisfying this objectivity condition on the application of the normative principle is a lack of empirical data (ibid., p. 9). But our conclusion implies the obstacle is greater-that the objectivity condition cannot be met, nor the principle applied, no matter the state of empirical data.

${ }^{1}$ Philip Kitcher, Science, Truth, and Democracy. (New York, NY: Oxford University Press, 2001).

${ }^{2}$ Evidence for the age of the putative clade is discussed in A. Becker and G. Theissen, "The major clades of MADS-box genes and their role in the development and evolution of flowering plants," Molecular Phylogenetics and Evolution XXIX (2003): 464-89.

${ }^{3}$ Carrie Morjan and Loren Rieseberg, "How species evolve collectively: implications of gene flow and selection for the spread of advantageous alleles," Molecular Ecology XIII (2004): 1341-56.

${ }^{4}$ E.g., p. 77 in David A. Baum, "Species as ranked taxa", Systematic Biology LVIII (2009): 74-86. 
${ }^{5}$ John Dupré, The Disorder of Things: Metaphysical Foundation of the Disunity of Science (Cambridge, MA: Harvard University Press, 1993); Marc Ereshefsky, "Eliminative Pluralism", Philosophy of Science LIX (1992): 671-690; Marc Ereshefsky, "Species Pluralism and Anti-Realism", Philosophy of Science LXV (1998): 103-120; Kitcher, op. cit; and Philip Kitcher, "Species", Philosophy of Science LI (1984): 308333.

${ }^{6}$ Ereshefsky, op. cit; cf. Joseph LaPorte, Joseph, "Is there a single objective, evolutionary tree of life?," The Journal of Philosophy, CII (2005): 357-74.

7 Lisa Gannett, "Making Populations: Bounding Genes in Space and in Time," Philosophy of Science LXX (2003): 989-1001.

${ }^{8}$ Kitcher (2001), op. cit.

${ }^{9}$ Kitcher, ibid., Dupré, op. cit., Ereshefsky op. cit.

${ }^{10}$ For syngameons see Alan R. Templeton, "The Meaning of Species and Speciation: A Genetic Perspective", in D. Otte and J. A. Endler, eds., Speciation and Its Consequences (Sunderland, MA: Sinauer, 1989), pp. 3-27. And for higher taxa see: Matthew J. Barker and Robert A. Wilson, "Cohesion, Gene Flow, and the Nature of Species," The Journal of Philosophy CVII (2010): 61-79; Marc Ereshefsky, "Species, Higher Taxa, and the Units of Evolution", Philosophy of Science LVIII (1991): 84-101.

${ }^{11}$ D. Brooks and D. McLennan, The Nature of Diversity (Chicago, IL: University of Chicago Press, 2002).

${ }^{12}$ Barker and Wilson, op. cit; and Matthew J. Barker, "The Empirical Inadequacy of Species Cohesion by Gene Flow," Philosophy of Science LXXIV (2007): 654-665. 
${ }^{13}$ At p. 1987 in T. Barbará, G. Martinelli, M. F. Fay, S. J. Mayo, and C. Lexer, "Population differentiation and species cohesion in two closely related plants adapted to neotropical high-altitude 'inselbergs', Alcantarea imperialis and Alcantarea genicultata (Bromeliaceae)," Molecular Ecology XVI (2007): 1981-92.

${ }^{14}$ Morjan and Rieseberg, op. cit.

${ }^{15}$ See pp. 74-75 in Baum, op. cit.

${ }^{16}$ Barker and Wilson, op. cit.

17 Brent D. Mishler and Robert N. Brandon, "Individuality, Pluralism, and the Phylogenetic Species Concept," Biology \& Philosophy II (1987): 397-414; and Barker and Wilson, op. cit.

${ }^{18}$ Roberta Millstein, "The Concepts of Population and Metapopulation in Evolutionary Biology and Ecology," in M. A. Bell, D. J. Futuyma, W. F. Eanes, and J. S. Levinton, eds., Evolution Since Darwin: The First 150 Years (Sunderland, MA: Sinauer, 2010), pp. 6186.

${ }^{19}$ Peter Godfrey-Smith, Darwinian Populations and Natural Selection. (New York, NY: Oxford University Press, 2009).

${ }^{20}$ David A. Baum and Stacey D. Smith, Tree Thinking: An Introduction to Phylogenetic Biology (Greenwood Village, CO: Roberts and Company Publishers, 2012).

${ }^{21}$ Brent D. Mishler, "Getting Rid of Species?," in R. A. Wilson, ed., Species: New Interdisciplinary Essays (Cambridge, MA: MIT Press, 1999), pp. 307-315.

${ }^{22}$ Baum, op. cit;; Joel D. Velasco, "Species Concepts Should Not Conflict with Evolutionary History, but Often Do," Studies in the History and Philosophy of Biological 
and Biomedical Sciences, XXXIX (2008): 407-414; Joel D. Velasco, "Species, Genes, and the Tree of Life", British Journal for the Philosophy of Science LXI (2010): 599-619. ${ }^{23}$ Velasco (2008), op. cit. ${ }^{24}$ Joel D. Velasco, "The Future of Systematics: Tree-Thinking Without the Tree," (submitted).

${ }^{25}$ Peter R. Grant, and B. Rosemary Grant, How and Why Species Multiply: The Radiation of Darwin's Finches. (Princeton, NJ: Princeton University Press, 2008). ${ }^{26}$ Kevin de Queiroz and Michael Donoghue, "Phylogenetic systematics or Nelson's version of Cladistics?," Cladistics VI (1990): 61-75.

${ }^{27}$ David A. Baum and Kerry L. Shaw, "Genealogical perspectives on the species Problem", in P. C. Hoch and A. G. Stephenson, eds., Experimental and Molecular Approaches to Plant Biosystematics (St. Louis, MO: Missouri Botanical Garden, 1995), pp. 289-303; Joel D. Velasco, "When monophyly is not enough: Exclusivity as the key to defining a phylogenetic species concept," Biology \& Philosophy XXIV (2009): 47386.

${ }^{28}$ Velasco (2010), op. cit. ${ }^{29}$ Olga Zhaxybayeva, Kristen S. Swithers, Pascal Lapierre, Gregory P. Fournier, Derek M. Bickhart, Robert T. DeBoy, Karen E. Nelson, Camilla L. Nesbø, W. Ford Doolittle, J. Peter Gogarten, and Kenneth M. Noll, "On the chimeric nature, thermophilic origin, and phylogenetic placement of the Thermotogales", PNAS CVI (2009): 5865-70. ${ }^{30}$ See Richard Boyd, "Homeostasis, Species and Higher Taxa," in Robert A. Wilson, ed., Species: New Interdisciplinary Essays (Cambridge, MA: MIT Press, 1999), pp. 141-185; and Robert A. Wilson, Matthew J. Barker, and Ingo Brigandt, "When Traditional 
Essentialism Fails: Biological Natural Kinds," Philosophical Topics XXXV (2007): 189216.

${ }^{31}$ D. Thomas and D. Gray, "Update COSEWIC Status Report on the Woodland Caribou Rangifer tarandus caribou in Canada," Committee on the Status of Endangered Wildlife in Canada (Ottawa, 2002). 